\title{
A SPLITTER THEOREM RELATIVE TO A FIXED BASIS
}

\author{
NICK BRETTELL AND CHARLES SEMPLE
}

\begin{abstract}
A standard matrix representation of a matroid $M$ represents $M$ relative to a fixed basis $B$, where contracting elements of $B$ and deleting elements of $E(M)-B$ correspond to removing rows and columns of the matrix, while retaining standard form. If $M$ is 3 -connected and $N$ is a 3 -connected minor of $M$, it is often desirable to perform such a removal while maintaining both 3-connectivity and the presence of an $N$-minor. We prove that, subject to a mild essential restriction, when $M$ has no 4-element fans with a specific labelling relative to $B$, there are at least two distinct elements, $s_{1}$ and $s_{2}$, such that for each $i \in\{1,2\}$, $\operatorname{si}\left(M / s_{i}\right)$ is 3-connected and has an $N$-minor when $s_{i} \in B$, and $\operatorname{co}\left(M \backslash s_{i}\right)$ is 3-connected and has an $N$-minor when $s_{i} \in E(M)-B$. We also show that if $M$ has precisely two such elements and $P$ is the set of elements that, when removed in the appropriate way, retain the $N$-minor, then $(P, E(M)-P)$ is a sequential 3-separation.
\end{abstract}

\section{INTRODUCTION}

Two classical results in matroid theory are Tutte's Wheels-and-Whirls Theorem [7] and Seymour's Splitter Theorem [6]. For a 3-connected matroid $M$ that is neither a wheel nor a whirl, the former says that $M$ has a singleelement deletion or a single-element contraction that is 3-connected. The latter strengthens this result and says that if $N$ is a 3 -connected minor of $M$, then $M$ has a single-element deletion or a single-element contraction that is 3 -connected with an $N$-minor. These theorems are important tools that enable inductive arguments to be made for 3-connected matroids in order to derive matroid structure results.

In the context of matroid representation theory, it is common practice to work with a standard matrix representation; that is, a representation $\left[I_{r} \mid D\right]$ where the columns of $I_{r}$ correspond to a basis $B$ of $M$. In this case, deleting an element of $E(M)-B$ corresponds to removing a column of the matrix, and contracting an element of $B$ corresponds to removing a row and column; both operations maintain a matrix in standard form. When an element is removed in this way, we say it is removed relative to $B$.

Date: September 6, 2012.

1991 Mathematics Subject Classification. 05B35.

Key words and phrases. Matroid representation, Wheels and Whirls Theorem, Splitter Theorem.

The first author was supported by a University of Canterbury Doctoral Scholarship and the second author was supported by the New Zealand Marsden Fund. 
Moreover, when removing elements in any other way, a pivot operation needs to be performed to remain in standard form, which can result in the loss of visible information in the original representation. In this paper we prove an analogue of the Splitter Theorem where elements are removed relative to a fixed basis.

Let $M$ be a 3-connected matroid, let $B$ be a basis of $M$, and let $N$ be a 3 -connected minor of $M$. We say that an element $e \in E(M)$ is $(N, B)$-robust if either

(i) $e \in B$ and $M / e$ has an $N$-minor, or

(ii) $e \in E(M)-B$ and $M \backslash e$ has an $N$-minor.

Oxley et al. 4] proved that $M$ has an element that can be removed relative to $B$ where the resulting matroid maintains 3-connectivity and retains an $N$-minor, provided $M$ has no 4-element fans and has an element that is $(N, B)$-robust. They demonstrated that the presence of an $(N, B)$-robust element is necessary, as there exist $M, N$, and $B$ such that $M$ has no $(N, B)$-robust elements.

Whittle and Williams [9] extended this result in one direction when considering 3-connectivity up to simplification or cosimplification. Following their example, we say that an element $e \in E(M)$ is removable with respect to $B$ if either

(i) $e \in B$ and $\operatorname{si}(M / e)$ is 3 -connected, or

(ii) $e \in E(M)-B$ and $\operatorname{co}(M \backslash e)$ is 3 -connected.

Whittle and Williams [9] proved that when $|E(M)| \geq 4$, the matroid $M$ has at least four elements that are removable with respect to $B$. This result is an analogue of the Wheels and Whirls Theorem [7].

In this paper we prove Theorem 1.1, an extension of the result by Oxley et al. 4]. We say an element $e \in E(M)$ is $(N, B)$-strong if either

(i) $e \in B$, and $\operatorname{si}(M / e)$ is 3 -connected and has an $N$-minor, or

(ii) $e \in E(M)-B$, and $\operatorname{co}(M \backslash e)$ is 3 -connected and has an $N$-minor.

Theorem 1.1. Let $M$ be a 3-connected matroid with no 4-element fans such that $|E(M)| \geq 5$, let $N$ be a 3-connected minor of $M$, and let $B$ be a basis of $M$. If $M$ has two distinct $(N, B)$-robust elements, then $M$ has two distinct $(N, B)$-strong elements.

This result resolves Whittle and Williams' conjecture [9, Conjecture 6.1]. It is worth noting that the theorem differs from the conjecture in that $M$ is required to have at least five elements, and two distinct $(N, B)$-robust elements. These are both necessary assumptions. To see that $M$ must have at least five elements, consider the matroid $U_{2,4}$ with minor $U_{1,3}$ or $U_{2,3}$. Furthermore, we give an example, in Section 5, of a 3-connected matroid with a 3 -connected proper minor $N$ that has only one $(N, B)$-robust element.

The requirement that $M$ has no 4-element fans is consistent with the work of Oxley et al. 4 and Whittle and Williams [9, but is not strictly necessary when taking into account which elements of the fan are in the basis $B$. 
Indeed, we prove a stronger result, Theorem 4.9, which demonstrates that $M$ still has the two desired elements when a 4-element fan is present, unless the fan has a particular labelling relative to $B$. In Section 5 we give an example illustrating that, when a matroid has a 4-element fan with this particular labelling, we cannot guarantee the presence of even a single $(N, B)$-strong element.

Having established a lower bound on the number of strong elements, it is natural to consider what can be said about matroids that have the minimum number of such elements. An exactly 3 -separating partition $(X, Y)$ of $M$ is sequential if there is an ordering $\left(e_{1}, e_{2}, \ldots, e_{k}\right)$ of $X$ or $Y$ such that $\left\{e_{1}, e_{2}, \ldots, e_{i}\right\}$ is 3 -separating for all $i \in\{1,2, \ldots, k\}$. A matroid has path-width three if its ground set is sequential; that is, there is an ordering $\left(e_{1}, e_{2}, \ldots, e_{n}\right)$ of $E(M)$ such that $\left\{e_{1}, e_{2}, \ldots, e_{i}\right\}$ is 3 -separating for all $i \in\{1,2, \ldots, n\}$. Whittle and Williams [9] proved that a matroid with precisely four removable elements with respect to some fixed basis has pathwidth three. In the latter part of the paper, we prove the following theorem.

Theorem 1.2. Let $M$ be a 3-connected matroid with no 4-element fans such that $|E(M)| \geq 5$, let $N$ be a 3-connected minor of $M$, and let $B$ be a basis of $M$. Let $P$ denote the set of $(N, B)$-robust elements of $M$. If $M$ has precisely two $(N, B)$-strong elements, then $(P, E(M)-P)$ is a sequential 3-separation.

As with Theorem 1.1, we are able to generalise Theorem 1.2 by considering the 4-element fans' labellings relative to the fixed basis. The stronger result is presented as Theorem 6.5 .

The paper is structured as follows. The next section contains some necessary preliminaries regarding connectivity and fans. In Section 3, we find the minimum number of removable elements in the absence of 4 -element fans that have either of two particular labellings relative to a fixed basis. In Section 4, we restrict our view to removable elements that also retain a copy of a specified minor, culminating in Theorem 4.9, a generalisation of Theorem 1.1. In Section 5, we give two examples illustrating the necessity of the conditions in the statement of the theorems. Finally, in Section 6, we prove Theorem 6.5, a generalisation of Theorem 1.2

The notation and terminology in the paper follow Oxley [2]. We write $x \in \mathrm{cl}^{(*)}(Y)$ to denote that either $x \in \operatorname{cl}(Y)$ or $x \in \operatorname{cl}^{*}(Y)$. The phrase by orthogonality refers to the fact that a circuit and a cocircuit cannot intersect in exactly one element. Lastly, we remark that the 3-connectivity conclusions in the theorems of this paper are up to parallel and series classes. However, with the help of Lemma 2.7 in the next section, it is easily seen that these conclusions are really up to parallel and series couples, where a parallel couple (respectively, series couple) is a parallel (respectively, series) class of size two. 


\section{Preliminaries}

Connectivity. Let $M$ be a matroid with ground set $E$. The connectivity function of $M$, denoted by $\lambda_{M}$, is defined on all subsets $X$ of $E$ by

$$
\lambda_{M}(X)=r(X)+r(E-X)-r(M) .
$$

A subset $X$ or a partition $(X, E-X)$ of $E$ is $k$-separating if $\lambda_{M}(X) \leq$ $k-1$. A $k$-separating partition $(X, E-X)$ is a $k$-separation if $|X| \geq k$ and $|E-X| \geq k$. A $k$-separating set $X$, a $k$-separating partition $(X, E-X)$ or a $k$-separation $(X, E-X)$ is exact if $\lambda_{M}(X)=k-1$. The matroid $M$ is $n$-connected if, for all $k<n$, it has no $k$-separations. When a matroid is 2-connected, we simply say it is connected.

The following lemma is a consequence of the easily verified fact that the connectivity function is submodular. The subsequent corollary follows by a routine induction argument.

Lemma 2.1. Let $M$ be a 3-connected matroid, and let $X$ and $Y$ be 3separating subsets of $E(M)$.

(i) If $|X \cap Y| \geq 2$, then $X \cup Y$ is 3-separating.

(ii) If $|E(M)-(X \cup Y)| \geq 2$, then $X \cap Y$ is 3-separating.

Corollary 2.2. Let $M$ be a 3-connected matroid, and let $\mathcal{X}$ be a finite set of 3-separating subsets of $E(M)$. If $\left|E(M)-\left(\bigcup_{X \in \mathcal{X}} X\right)\right| \geq 2$, then $\bigcap_{X \in \mathcal{X}} X$ is 3-separating.

The following two lemmas are used frequently in the paper. The first is well-known (see, for example, [2, Proposition 2.1.12]) and is a consequence of orthogonality; the second is a consequence of the first.

Lemma 2.3. Let $e$ be an element of a matroid $M$, and let $X$ and $Y$ be disjoint sets whose union is $E(M)-\{e\}$. Then $e \in \operatorname{cl}(X)$ if and only if $e \notin \operatorname{cl}^{*}(Y)$.

Lemma 2.4. Let $X$ be an exactly 3-separating set in a 3-connected matroid $M$, and suppose that $e \in E(M)-X$. Then $X \cup\{e\}$ is 3-separating if and only if $e \in \mathrm{cl}^{(*)}(X)$. Moreover, $X \cup\{e\}$ is exactly 3-separating if and only if $e$ is in exactly one of $\operatorname{cl}(X) \cap \operatorname{cl}(E(M)-X-\{e\})$ and $\operatorname{cl}^{*}(X) \cap \operatorname{cl}^{*}(E(M)-X-\{e\})$.

A $k$-separation $(X, E-X)$ of a matroid $M$ with ground set $E$ is vertical if $r(X) \geq k$ and $r(E-X) \geq k$. We also say a partition $(X,\{e\}, Y)$ of $E$ is a vertical 3-separation when $(X \cup\{e\}, Y)$ and $(X, Y \cup\{e\})$ are both vertical 3 -separations and $e \in \operatorname{cl}(X) \cap \operatorname{cl}(Y)$. The next three lemmas will be used frequently; a proof of the first is in [4, the second follows from a result established in [3], while the third is elementary.

Lemma 2.5. Let $M$ be a 3 -connected matroid and let $z \in E(M)$. If $\operatorname{si}(M / z)$ is not 3-connected, then $M$ has a vertical 3-separation $(X,\{z\}, Y)$.

Lemma 2.6. Let $(X,\{z\}, Y)$ be a vertical 3-separation of a 3-connected matroid $M$. Then there exists a vertical 3-separation $\left(X^{\prime},\{z\}, Y^{\prime}\right)$ such that $X^{\prime} \subseteq X$, and $Y^{\prime} \cup\{z\}$ is closed. 
Lemma 2.7. Let $M$ be a 3-connected matroid and let $L$ be a rank-2 subset with at least four elements. If $l \in L$, then $M \backslash l$ is 3-connected.

The next four lemmas are more technical; proofs for the first three are given elsewhere. The first will be referred to as Bixby's Lemma [1] the second is due to Whittle and Williams [9]; and the third was proved by Whittle [8].

Lemma 2.8. Let $e$ be an element of a 3-connected matroid $M$. Then either $\operatorname{si}(M / e)$ or $\operatorname{co}(M \backslash e)$ is 3-connected.

A segment in a matroid $M$ is a subset $L$ of $E(M)$ such that $M \mid L \cong U_{2, k}$ for some $k \geq 2$, while a cosegment of $M$ is a segment of $M^{*}$.

Lemma 2.9. Let $M$ be a 3-connected matroid with a triad $\{a, b, c\}$ and $a$ circuit $\{a, b, c, d\}$. Then at least one of the following holds:

(i) either $\operatorname{co}(M \backslash a)$ or $\operatorname{co}(M \backslash c)$ is 3-connected, or

(ii) there exist elements $a^{\prime}, c^{\prime} \in E(M)$ such that $\left\{a, a^{\prime}, b\right\}$ and $\left\{b, c, c^{\prime}\right\}$ are triangles, or

(iii) there exists an element $z \in E(M)-\{a, b, c, d\}$ such that $\{a, b, c, z\}$ is a cosegment.

Lemma 2.10. Let $C^{*}$ be a rank-3 cocircuit of a 3-connected matroid $M$. If $e \in C^{*}$ has the property that $\mathrm{cl}_{M}\left(C^{*}\right)-\{e\}$ contains a triangle of $M / e$, then $\operatorname{si}(M / e)$ is 3-connected.

Lemma 2.11. Let $(X, Y)$ be a 3-separation of a 3-connected matroid $M$. If $X \cap \operatorname{cl}(Y) \neq \emptyset$ and $X \cap \operatorname{cl}^{*}(Y) \neq \emptyset$, then $|X \cap \operatorname{cl}(Y)|=1$ and $\left|X \cap \operatorname{cl}^{*}(Y)\right|=1$.

Proof. Let $x \in X \cap \mathrm{cl}^{*}(Y)$, and consider $M \backslash x$. Since $x \in \operatorname{cl}^{*}(Y)$, it follows by Lemma 2.3 that $x \notin \operatorname{cl}(X-\{x\})$. Therefore, as $M$ is 3 -connected,

$$
r(X-\{x\})+r(Y)-r(M \backslash x)=r(X)-1+r(Y)-r(M)=1,
$$

and so $(X-\{x\}, Y)$ is a 2-separation of $M \backslash x$. Hence, as $M$ has no 2-circuits, $|X \cap \operatorname{cl}(Y)| \leq 1$. Thus $|X \cap \operatorname{cl}(Y)|=1$.

Now suppose that $\left|X \cap \operatorname{cl}^{*}(Y)\right| \geq 2$, and let $x^{\prime} \in X \cap \mathrm{cl}^{*}(Y)$ such that $x^{\prime} \neq x$. Then, as $x \in \mathrm{cl}^{*}(Y)$ and $x^{\prime} \in \mathrm{cl}^{*}(Y \cup\{x\})$, it follows by Lemma 2.3 that $x \notin \operatorname{cl}(X-\{x\})$ and $x^{\prime} \notin \operatorname{cl}\left(X-\left\{x, x^{\prime}\right\}\right)$. Thus, as $M$ is 3-connected,

$$
r\left(X-\left\{x, x^{\prime}\right\}\right)+r(Y)-r\left(M \backslash\left\{x, x^{\prime}\right\}\right)=r(X)-2+r(Y)-r(M)=0 .
$$

In particular, $X-\left\{x, x^{\prime}\right\}$ is a separator of $M \backslash\left\{x, x^{\prime}\right\}$. But $X \cap \operatorname{cl}(Y)$ is nonempty in $M$ and contains neither $x$ nor $x^{\prime}$; otherwise, $M$ has a 2-separation. Therefore $\left(X-\left\{x, x^{\prime}\right\}\right) \cap \operatorname{cl}(Y)$ is non-empty in $M \backslash\left\{x, x^{\prime}\right\}$; a contradiction. Hence $\left|X \cap \operatorname{cl}^{*}(Y)\right|=1$, completing the proof of the lemma.

Fans. Let $M$ be a 3 -connected matroid. A subset $F$ of $E(M)$ having at least three elements is a fan if there is an ordering $\left(f_{1}, f_{2}, \ldots, f_{k}\right)$ of the elements of $F$ such that 
(i) for all $i \in\{1,2, \ldots, k-2\}$, the triple $\left\{f_{i}, f_{i+1}, f_{i+2}\right\}$ is either a triangle or a triad, and

(ii) for all $i \in\{1,2, \ldots, k-3\}$, if $\left\{f_{i}, f_{i+1}, f_{i+2}\right\}$ is a triangle, then $\left\{f_{i+1}, f_{i+2}, f_{i+3}\right\}$ is a triad, while if $\left\{f_{i}, f_{i+1}, f_{i+2}\right\}$ is a triad, then $\left\{f_{i+1}, f_{i+2}, f_{i+3}\right\}$ is a triangle.

An ordering of $F$ satisfying (i) and (ii) is a fan ordering of $F$. If $F$ has a fan ordering $\left(f_{1}, f_{2}, \ldots, f_{k}\right)$ where $k \geq 4$, then $f_{1}$ and $f_{k}$ are the ends of $F$, and $f_{2}, f_{3}, \ldots, f_{k-1}$ are the internal elements of $F$.

Let $F$ be a fan with ordering $\left(f_{1}, f_{2}, \ldots, f_{k}\right)$ where $k \geq 5$, and let $i \in$ $\{1,2, \ldots, k\}$. An element $f_{i}$ is a spoke element of $F$ if $\left\{f_{1}, f_{2}, f_{3}\right\}$ is a triangle and $i$ is odd, or if $\left\{f_{1}, f_{2}, f_{3}\right\}$ is a triad and $i$ is even; otherwise $f_{i}$ is a rim element. For a fan $F$ with ordering $\left(f_{1}, f_{2}, f_{3}, f_{4}\right)$, the element $f_{1}$ is a spoke element of $F$ if $\left\{f_{1}, f_{2}, f_{3}\right\}$ is a triangle, otherwise it is a rim element; while $f_{4}$ is a spoke element if $\left\{f_{1}, f_{2}, f_{3}\right\}$ is a triad, otherwise it is a rim element.

The next lemma is a variant on a well-known result, which follows easily from Bixby's Lemma.

Lemma 2.12. Let $M$ be a 3-connected matroid such that $|E(M)| \geq 7$. Suppose $M$ has a fan $F$ of at least four elements, and let $f$ be an end of $F$.

(i) If $f$ is a spoke element, then $\operatorname{co}(M \backslash f)$ is 3 -connected and $\operatorname{si}(M / f)$ is not 3-connected.

(ii) If $f$ is a rim element, then $\operatorname{si}(M / f)$ is 3-connected and $\operatorname{co}(M \backslash f)$ is not 3-connected.

In what follows, a key difference from the prior work of Oxley et al. and Whittle and Williams is that we relax the assumption that no 4-element fans are present. However, a 4-element fan with one of two particular labellings, relative to a fixed basis, requires special attention. We now define these fans.

Let $M$ be a matroid and let $B$ be a basis of $M$. We define a Type I fan relative to $B$ in $M$ as a 4 -element fan $F$ with ordering $\left(f_{1}, f_{2}, f_{3}, f_{4}\right)$ where $\left\{f_{1}, f_{2}, f_{3}\right\}$ is a triangle and $F \cap B=\left\{f_{1}, f_{3}\right\}$. We define a Type II fan relative to $B$ in $M$ as a 4 -element fan $F$ with ordering $\left(f_{1}, f_{2}, f_{3}, f_{4}\right)$ where $\left\{f_{1}, f_{2}, f_{3}\right\}$ is a triangle and $F \cap B=\left\{f_{1}, f_{3}, f_{4}\right\}$.

\section{The Existence of Removable Elements}

In this section we prove Lemma 3.4, which will turn out to be the crux of the proofs of Theorem 1.1 and Theorem 1.2 .

Lemma 3.1. Let $M$ be a 3-connected matroid with $r(M) \geq 4$. Suppose that $C^{*}$ is a rank-3 cocircuit of $M$ such that $\left|C^{*}\right| \geq 4$.

(i) If there is no $T \subseteq C^{*}$ such that $T$ is a triangle, then $\operatorname{co}(M \backslash d)$ is 3 -connected for all $d \in C^{*}$.

(ii) If $T \subseteq C^{*}$ such that $T$ is a triangle, then $\operatorname{co}(M \backslash d)$ is 3 -connected for all $d \in T$. 
Proof. Suppose $\operatorname{co}(M \backslash d)$ is not 3-connected for some $d$ satisfying the hypothesis of either (i) or (ii). Then $M \backslash d$ has a 2-separation $(U, V)$ in which neither $U$ nor $V$ is a series class. Clearly, $d \notin \operatorname{cl}(U)$ and $d \notin \operatorname{cl}(V)$; otherwise, $M$ has a 2-separation. Thus $U \cap C^{*}$ and $V \cap C^{*}$ are both non-empty. Furthermore, either $U$ or $V$ contains two distinct elements $x_{1}, x_{2} \in C^{*}$ such that $C^{*} \subseteq \operatorname{cl}\left(\left\{x_{1}, x_{2}, d\right\}\right)$. Without loss of generality, we may assume that $\left\{x_{1}, x_{2}\right\} \subseteq U$. The set $U \cup\{d\}$ is exactly 3 -separating. Therefore, by repeated applications of Lemma 2.4, for each subset $D$ of $V \cap C^{*}$, the set $D \subseteq \operatorname{cl}(V-D)$ provided $|V-D| \geq 2$. Let $H=E(M)-C^{*}$. If $|V \cap H| \geq 2$, then $\operatorname{cl}(H) \cap C^{*}$ is non-empty, contradicting the fact that $H$ is a hyperplane. Thus $|V \cap H| \leq 1$. If $|V \cap H|=0$, then $H \subseteq U$ and so, as $U \cap C^{*}$ is nonempty, $r(U)=r(M)$. This implies that $V$ is a parallel class; a contradiction as $M$ is 3-connected. Hence $|V \cap H|=1$ and $r(V)=2$. Let $V \cap H=\{h\}$. If $\left|V \cap C^{*}\right| \geq 2$, then $h \in \operatorname{cl}\left(C^{*}\right)$ and so, by Lemma 2.4, $h \in \operatorname{cl}(H-\{h\})$. In particular, $H \subseteq \mathrm{cl}(U)$ and so $r(U)=r(M)$; a contradiction. Therefore $\left|V \cap C^{*}\right|=1$, and so $V$ is a 2-element cocircuit, a contradiction. This completes the proof of the lemma.

Let $M$ be a matroid with a basis $B$ where there exists an element $b \in B$ that is not removable with respect to $B$, and let $(X,\{b\}, Y)$ be a vertical 3 -separation of $M$. We say that $X \cup\{b\}$ is minimal in $(X,\{b\}, Y)$ when, for every $b_{z} \in X \cap B$ such that $\operatorname{si}\left(M / b_{z}\right)$ is not 3-connected, a vertical 3-separation $\left(X_{z},\left\{b_{z}\right\}, Y_{z}\right)$ has neither $X_{z}$ nor $Y_{z}$ contained in $X \cup\{b\}$.

The next lemma is extracted from proofs by both Oxley et al. 4, Lemma 3.2], and Whittle and Williams [9, Lemma 3.1].

Lemma 3.2. Let $M$ be a 3-connected matroid with a basis B. Suppose there exists an element $b_{1} \in B$ that is not removable with respect to $B$, and let $\left(X_{1},\left\{b_{1}\right\}, Y_{1}\right)$ be a vertical 3-separation of $M$ such that $Y_{1} \cup\left\{b_{1}\right\}$ is closed and $X_{1} \cup\left\{b_{1}\right\}$ is minimal in $\left(X_{1},\left\{b_{1}\right\}, Y_{1}\right)$. If $\left(X_{2},\left\{b_{2}\right\}, Y_{2}\right)$ is a vertical 3-separation of $M$ with $Y_{2} \cup\left\{b_{2}\right\}$ closed, $b_{2} \in X_{1} \cap B$ and $b_{1} \in Y_{2}$, then all of the following hold:

(i) $X_{1} \cap X_{2}, X_{1} \cap Y_{2}, Y_{1} \cap X_{2}$ and $Y_{1} \cap Y_{2}$ are all non-empty,

(ii) $r\left(\left(X_{1} \cap X_{2}\right) \cup\left\{b_{2}\right\}\right)=2$, and

(iii) if $\left|Y_{1} \cap X_{2}\right| \geq 2$, then $r\left(\left(X_{1} \cap Y_{2}\right) \cup\left\{b_{1}, b_{2}\right\}\right)=2$.

The next lemma is straightforward, but is used frequently in the proof of Lemma 3.4 .

Lemma 3.3. Let $M$ be a matroid with distinct elements $f_{1}, f_{2}, f_{3}$ and $f_{4}$. If the only triangle containing $f_{3}$ is $\left\{f_{1}, f_{2}, f_{3}\right\}$ and the only triad containing $f_{2}$ is $\left\{f_{2}, f_{3}, f_{4}\right\}$, then $\operatorname{si}\left(M / f_{3}\right) \cong \operatorname{co}\left(M \backslash f_{2}\right)$.

Lemma 3.4. Let $M$ be a 3-connected matroid and let $B$ be a basis of $M$. Suppose $M$ has no Type I fans relative to $B$, suppose there exists an element $b \in B$ such that $\operatorname{si}(M / b)$ is not 3-connected, and let $(X,\{b\}, Y)$ be a vertical 3-separation of $M$. Then one of the following holds: 
(i) there exist distinct elements $s_{1}, s_{2} \in X$ that are removable with respect to $B$, or

(ii) there exist distinct removable elements $s_{1} \in X$ and $s_{2} \in \mathrm{cl}^{*}(X) \cap B$, and a vertical 3-separation $\left(X^{\prime},\left\{b^{\prime}\right\}, Y^{\prime}\right)$ of $M$ such that $X^{\prime} \cup\left\{s_{2}\right\}$ is a 4-element cosegment containing $s_{1}$, the element $b^{\prime} \in B$ is not removable with respect to $B$, and $X^{\prime} \cup\left\{b^{\prime}\right\} \subseteq X \cup\{b\}$, or

(iii) there exist distinct elements $s_{1} \in X$ and $s_{2}, s_{3} \in \operatorname{cl}(X) \cap(E(M)-B)$ that are removable with respect to $B$, or

(iv) $M$ has a Type II fan relative to $B$ contained in $X \cup\{b\}$.

Proof. By Lemma 2.6, there exists a vertical 3-separation $\left(X^{\prime},\{b\}, Y^{\prime}\right)$ such that $Y^{\prime} \cup\{b\}$ is closed and $X^{\prime} \subseteq X$. If the lemma holds for the vertical 3 -separation $\left(X^{\prime},\{b\}, Y^{\prime}\right)$, then clearly it holds for the vertical 3-separation $(X,\{b\}, Y)$; so we may assume that $Y \cup\{b\}$ is closed. Note that $|X \cap B| \geq 1$. If $X \cap B$ contains two or more elements that are removable with respect to $B$, then (i) holds; so we assume this is not the case. As a result, it is sufficient to consider the following two cases:

(I) $X \cap B=\left\{b_{c}\right\}$ and $b_{c}$ is removable with respect to $B$, or

(II) there exists an element $b_{x} \in X \cap B$ such that $b_{x}$ is not removable with respect to $B$.

We first show that in case (I), one of (i), (ii) or (iii) holds. More generally, we prove the following:

3.4.1. If there exists a vertical 3 -separation $\left(X_{1},\left\{b_{1}\right\}, Y_{1}\right)$ such that $X_{1} \cap B=$ $\left\{b_{c}\right\}$, where $b_{c}$ is removable with respect to $B$, the element $b_{1} \in B$ is not removable with respect to $B$, and $X_{1} \cup\left\{b_{1}\right\} \subseteq X \cup\{b\}$, then (i), (ii) or (iii) holds.

Since $\left|X_{1} \cap B\right|=1$ and $Y_{1} \cup\left\{b_{1}\right\}$ is closed, $Y_{1} \cup\left\{b_{1}\right\}$ is a hyperplane and $X_{1}$ is a rank-3 cocircuit. If $\left|X_{1}\right| \geq 4$, then, by Lemma 3.1, there exists a removable element in $X_{1} \cap(E(M)-B)$, so (i) holds. Thus, assume that $\left|X_{1}\right|=3$, and let $X_{1}=\left\{a, b_{c}, c\right\}$. If $a$ or $c$ is removable with respect to $B$, then (i) is satisfied, so we may also assume neither $\operatorname{co}(M \backslash a) \operatorname{nor} \operatorname{co}(M \backslash c)$ is 3-connected.

Suppose that $X_{1} \cup\left\{b_{1}\right\}$ is not a 4-element fan. Then $X_{1} \cup\left\{b_{1}\right\}$ is a circuit. As neither $\operatorname{co}(M \backslash a)$ nor $\operatorname{co}(M \backslash c)$ is 3-connected, it follows by Lemma 2.9 that either $\left\{a, b_{c}, c\right\}$ are the internal elements of a 5-element fan, or there exists an element $z \in E(M)-\left(X_{1} \cup\left\{b_{1}\right\}\right)$ such that $X_{1} \cup\{z\}$ is a cosegment. If the latter, then (ii) holds by the dual of Lemma 2.7. If the former, then both ends of the 5-element fan, $a^{\prime}$ and $c^{\prime}$ say, are in $E(M)-B$, otherwise we have a Type I fan. It follows, by Lemma 2.12, that $a^{\prime}$ and $c^{\prime}$ are both removable. Since $b_{c} \in X$ is also removable, (iii) holds.

Now consider the case where $X_{1} \cup\left\{b_{1}\right\}$ is a 4-element fan. Then, up to relabelling, either $\left\{a, b_{c}, b_{1}\right\}$ or $\left\{a, c, b_{1}\right\}$ is a triangle. If $\left\{a, b_{c}, b_{1}\right\}$ is a triangle, then $X_{1} \cup\left\{b_{1}\right\}$ is a Type I fan; a contradiction. Thus $\left\{a, c, b_{1}\right\}$ is a triangle. If $c$ is contained in a triad $T^{*}$ that is not $\left\{a, b_{c}, c\right\}$, then, by 
orthogonality, $T^{*}$ contains either $a$ or $b_{1}$. But if it contains $a$, then $X_{1} \cup T^{*}$ is a cosegment of four elements, so by the dual of Lemma 2.7, (ii) holds. If instead $\left\{b_{1}, c\right\}$ is contained in $T^{*}$, then $a$ is a spoke and an end element of a 4-element fan, so $\operatorname{co}(M \backslash a)$ is 3-connected by Lemma 2.12 a contradiction. It follows that the only triad containing $c$ is $\left\{a, b_{c}, c\right\}$.

If the only triangle containing $a$ is $\left\{a, c, b_{1}\right\}$, then $\operatorname{si}(M / a) \cong \operatorname{co}(M \backslash c)$ by Lemma 3.3, so $\operatorname{si}(M / a)$ is not 3-connected. But $\operatorname{co}(M \backslash a)$ is not 3-connected, contradicting Bixby's Lemma, so $a$ is contained in a triangle other than $\left\{a, c, b_{1}\right\}$. By orthogonality, such a triangle contains either $\left\{a, b_{c}\right\}$ or $\{a, c\}$, but the latter is not possible since $\left\{a, c, b_{1}\right\}$ is a triangle and $Y_{1} \cup\left\{b_{1}\right\}$ is closed. So $a$ is contained in a triangle $\left\{a, b_{c}, a^{\prime}\right\}$ say. Since $\operatorname{si}\left(M / b_{c}\right)$ is 3connected but $\operatorname{co}(M \backslash a)$ is not 3 -connected, either $b_{c}$ is contained in a triangle other than $\left\{a, b_{c}, a^{\prime}\right\}$, or $a$ is contained in a triad other than $\left\{a, b_{c}, c\right\}$, by Lemma 3.3. But $\left\{b_{1}, c, a, b_{c}, a^{\prime}\right\}$ is a 5 -element fan and $r(M) \geq 4$, so, by [5, Lemma 3.4], the only triad containing $a$ is $\left\{a, b_{c}, c\right\}$. Thus, by orthogonality and since $Y_{1} \cup\left\{b_{1}\right\}$ is closed, $\left\{b_{c}, c\right\}$ is contained in a triangle $\left\{b_{c}, c, c^{\prime}\right\}$ say. Now $\left(a^{\prime}, a, b_{c}, c, c^{\prime}\right)$ is a fan ordering of a 5-element fan. The elements $a^{\prime}, c^{\prime} \in \operatorname{cl}(X)$ are both in $E(M)-B$, or this fan contains a Type I fan. It follows, by Lemma 2.12, that $a^{\prime}$ and $c^{\prime}$ are both removable, so (iii) holds, completing the proof of 3.4 .1 .

Now consider (II). We show that when 3.4.1 does not hold, we have the following:

3.4.2. There exists a vertical 3-separation $\left(X_{1},\left\{b_{1}\right\}, Y_{1}\right)$ such that $X_{1} \cup$ $\left\{b_{1}\right\} \subseteq X \cup\{b\}$, the subset $Y_{1} \cup\left\{b_{1}\right\}$ is closed, $X_{1} \cup\left\{b_{1}\right\}$ is minimal in $\left(X_{1},\left\{b_{1}\right\}, Y_{1}\right)$, and there exists an element $b_{2} \in X_{1} \cap B$ such that $\operatorname{si}\left(M / b_{2}\right)$ is not 3-connected.

If $X \cup\{b\}$ is minimal in $(X,\{b\}, Y)$, then 3.4 .2 holds. So assume there exists an element $b_{z} \in X \cap B$ such that $\operatorname{si}\left(M / b_{z}\right)$ is not 3 -connected, and a vertical 3-separation $\left(X_{z},\left\{b_{z}\right\}, Y_{z}\right)$ such that, without loss of generality, $X_{z} \subseteq\left(X-\left\{b_{z}\right\}\right) \cup\{b\}$. By Lemma 2.6 we can assume $Y_{z} \cup\left\{b_{z}\right\}$ is closed. Now, if $X_{z}=\left(X-\left\{b_{z}\right\}\right) \cup\{b\}$, then $Y_{z}=Y$, but this implies that $b_{z} \in$ $\operatorname{cl}\left(Y_{z}\right)=\operatorname{cl}(Y)$; a contradiction. So $X_{z} \varsubsetneqq\left(X-\left\{b_{z}\right\}\right) \cup\{b\}$. If $X_{z} \cup\left\{b_{z}\right\}$ is not already minimal in $\left(X_{z},\left\{b_{z}\right\}, Y_{z}\right)$, then, by repeating this procedure, we eventually obtain a vertical 3-separation $\left(X_{1},\left\{b_{1}\right\}, Y_{1}\right)$ where $X_{1} \cup\left\{b_{1}\right\}$ is minimal in $\left(X_{1},\left\{b_{1}\right\}, Y_{1}\right)$. If $X_{1} \cap B=\left\{b_{c}\right\}$ where $b_{c}$ is removable with respect to $B$, then 3.4 .1 holds, so the lemma holds. Otherwise, $X_{1} \cap B$ contains an element, $b_{2}$ say, that is not removable with respect to $B$.

By Lemma 2.5. $M$ has a vertical 3-separation $\left(X_{2},\left\{b_{2}\right\}, Y_{2}\right)$ where $b_{2} \in$ $X_{1}$. Without loss of generality, let $b_{1} \in Y_{2}$ where, due to Lemma 2.6, we can assume $Y_{2} \cup\left\{b_{2}\right\}$ is closed. By Lemma 3.2, each of $X_{1} \cap X_{2}, X_{1} \cap Y_{2}$, $Y_{1} \cap X_{2}$, and $Y_{1} \cap Y_{2}$ is non-empty, and $r\left(\left(X_{1} \cap X_{2}\right) \cup\left\{b_{2}\right\}\right)=2$. We consider two subcases: $\left|Y_{1} \cap X_{2}\right| \geq 2$, and $\left|Y_{1} \cap X_{2}\right|=1$.

3.4.3. The lemma holds when $\left|Y_{1} \cap X_{2}\right| \geq 2$. 
If $\left|Y_{1} \cap X_{2}\right| \geq 2$, then, by Lemma 3.2(iii), $r\left(\left(X_{1} \cap Y_{2}\right) \cup\left\{b_{1}, b_{2}\right\}\right)=2$. Let $L_{1}=\left(X_{1} \cap Y_{2}\right) \cup\left\{b_{1}\right\}$ and $L_{2}=\left(X_{1} \cap X_{2}\right) \cup\left\{b_{2}\right\}$. If $\left|L_{2}\right| \geq 4$, then, by Lemma 2.7, (i) holds. Similarly, if $\left|\operatorname{cl}\left(L_{1}\right)\right| \geq 4$, then $L_{1}$ contains at least two removable elements, and these elements are in $X_{1}$ since $Y_{1} \cup\left\{b_{1}\right\}$ is closed, thereby satisfying (i). Hence, since $X_{1} \cap Y_{2}$ is non-empty, we may assume $\left|\operatorname{cl}\left(L_{1}\right)\right|=3$ and $\left|L_{2}\right| \in\{2,3\}$.

Let $X_{1} \cap Y_{2}=\{a\}$ and $c \in X_{1} \cap X_{2}$. Note that $a \in E(M)-B$. If $\left|L_{2}\right|=2$, then $\left|X_{1} \cap X_{2}\right|=1, X_{1}=\left\{a, b_{2}, c\right\}$ is a triad and $\operatorname{cl}\left(L_{1}\right)=\left\{b_{1}, a, b_{2}\right\}$ is a triangle, so $\left\{b_{1}, a, b_{2}, c\right\}$ is a 4-element fan. If $c \in E(M)-B$, then $X_{1} \cup\left\{b_{1}\right\}$ is a Type I fan; a contradiction. But if $c \in B$, then $X_{1} \cup\left\{b_{1}\right\}$ is a Type II fan, in which case (iv) holds.

Now suppose $\left|L_{2}\right|=3$ and, in particular, $X_{1} \cap X_{2}=\{c, d\}$. Since $r\left(L_{2}\right)=$ 2 , we may assume without loss of generality that $d \in E(M)-B$. By Lemma 3.1(ii), $\operatorname{co}(M \backslash d)$ and $\operatorname{co}(M \backslash c)$ are 3-connected. If $c \in E(M)-B$, then (i) holds. Furthermore, if $c \in B$, then (i) also holds as $\operatorname{si}(M / c)$ is 3 -connected by Lemma 2.10. Thus 3.4 .3 holds.

It remains to prove that the lemma holds when $\left|Y_{1} \cap X_{2}\right|=1$. First, we show that, in such a situation, if there is an element of $B$ in $X_{1} \cap X_{2}$, then the lemma holds.

3.4.4. If, for some $b_{z} \in B$ such that $\mathrm{si}\left(M / b_{z}\right)$ is not 3-connected, $\left(X_{z},\left\{b_{z}\right\}, Y_{z}\right)$ is a vertical 3-separation of $M$ where $\left|Y_{1} \cap X_{z}\right|=1$ and there exists an element $p \in\left(X_{1} \cap X_{z}\right) \cap B$, then (i) holds.

By Lemma 3.2, $r\left(\left(X_{1} \cap X_{z}\right) \cup\left\{b_{z}\right\}\right)=2$, so if $\left|\left(X_{1} \cap X_{z}\right) \cup\left\{b_{z}\right\}\right| \geq 4$, then (i) holds by Lemma 2.7. So let $X_{1} \cap X_{z}=\{p, q\}$ where $p \in B$ and $q \in E(M)-B$, and let $Y_{1} \cap X_{z}=\{y\}$. First, suppose that $\operatorname{si}(M / p)$ is not 3-connected. Then, by Lemmas 2.5 and 2.6, there exists a vertical 3-separation $\left(X_{p},\{p\}, Y_{p}\right)$ such that $b_{1} \in Y_{p}$ and $Y_{p} \cup\{p\}$ is closed. By Lemma 3.2. $\left(X_{1} \cap X_{p}\right) \cup\{p\}$ is a rank-2 set, and if $\left|Y_{1} \cap X_{p}\right| \geq 2$, then $r\left(\left(X_{1} \cap Y_{p}\right) \cup\left\{b_{1}, p\right\}\right)=2$. If, indeed, $\left|Y_{1} \cap X_{p}\right| \geq 2$, then $r\left(X_{1}\right)=3$ and it follows, by Lemmas 2.10 and 3.1 (ii), that $p$ and $q$ are removable, satisfying (i). So assume that $\left|Y_{1} \cap X_{p}\right|=1$. Then $\left(X_{1} \cap X_{p}\right) \cup\{p\}$ is a rank-2 set of at least three elements. If this set has four or more elements, then (i) holds by Lemma 2.7, so assume $\left|X_{1} \cap X_{p}\right|=2$. Now $\left(X_{1} \cap X_{p}\right) \cup\{p\}$ is a triangle contained in $X_{1}$, but since $Y_{z} \cup\left\{b_{z}\right\}$ is closed, this triangle contains $q$. Then either $\left(X_{1} \cap X_{p}\right) \cup\left\{b_{z}, p\right\}$ is a rank-2 set of four elements, so (i) holds by Lemma 2.7, or $X_{1} \cap X_{p}=\left\{q, b_{z}\right\}$. Since $X_{p}$ is a triad, if $Y_{1} \cap X_{p}=\{y\}$, then $\left\{y, p, q, b_{z}\right\}$ is a 4-element cosegment, and $\operatorname{si}(M / p)$ is 3 -connected by the dual of Lemma 2.7; a contradiction. So $Y_{1} \cap X_{p}=\left\{y^{\prime}\right\}$ where $y^{\prime} \neq y$, and $\left\{y, y^{\prime}\right\} \subseteq \operatorname{cl}^{*}\left(X_{1}\right)$. But, recalling that $b_{1} \in \operatorname{cl}\left(X_{1}\right)$, this contradicts Lemma 2.11.

Now suppose that $\operatorname{si}(M / p)$ is 3-connected. If $\operatorname{co}(M \backslash q)$ is also 3-connected, then (i) holds, so assume this is not the case. Now, $\operatorname{si}(M / p) \nsubseteq \operatorname{co}(M \backslash q)$, so, by Lemma 3.3 , either $p$ is contained in a triangle other than $\left\{p, q, b_{z}\right\}$, or $q$ is contained in a triad other than $\{p, q, y\}$. Consider the former; by 
orthogonality and since $Y_{z} \cup\left\{b_{z}\right\}$ is closed, $\{p, y\}$ is contained in a triangle $T$. Let $T-\{p, y\}=\left\{y^{\prime}\right\}$. Note that $y \in B$, otherwise $X_{z} \cup\left\{b_{z}\right\}$ is a 4-element fan. Since $Y_{1} \cup\left\{b_{1}\right\}$ is closed, and due to the rank of $T, y^{\prime} \in X_{1} \cap(E(M)-B)$. By Lemma 2.12 $y^{\prime}$ is removable so (i) holds. Now consider when $q$ is in a triad $T^{*}$ other than $\{p, q, y\}$. By orthogonality, $T^{*}$ contains $p$ or $b_{z}$. If $\left\{q, b_{z}\right\}$ is contained in $T^{*}$, then $p$ is a spoke element and an end of a 4-element fan, so $\operatorname{si}(M / p)$ is not 3 -connected by Lemma 2.12, a contradiction. So assume that $\{p, q\}$ is contained in $T^{*}$. By Lemma 2.11, $T^{*} \subseteq X_{1}$. If $b_{z} \in \mathrm{cl}^{*}\left(T^{*}\right)$, then $b_{z}$ is removable by the dual of Lemma 2.7; a contradiction. It follows, by Lemma 2.3. that $\left(T^{*},\left\{b_{z}\right\}, E(M)-\left(T^{*} \cup\left\{b_{z}\right\}\right)\right)$ is a vertical 3-separation, contradicting that $X_{1} \cup\left\{b_{1}\right\}$ is minimal in $\left(X_{1},\left\{b_{1}\right\}, Y_{1}\right)$. Thus 3.4 .4 holds.

3.4.5. The lemma holds when $\left|Y_{1} \cap X_{2}\right|=1$.

As $\left|X_{2}\right| \geq 3$ and $b_{1} \notin X_{2}$, it follows that $\left|X_{1} \cap X_{2}\right| \geq 2$. By Lemma 3.2. $r\left(\left(X_{1} \cap X_{2}\right) \cup\left\{b_{2}\right\}\right)=2$. If $\left|X_{1} \cap X_{2}\right| \geq 3$, then (i) holds by Lemma 2.7 . Therefore we may assume that $\left|X_{1} \cap X_{2}\right|=2$. At most one element in $X_{1} \cap X_{2}$ is in $B$, but if there is precisely one such element, then (i) holds by 3.4 .4 . So let $X_{1} \cap X_{2}=\{p, q\}$, where $\{p, q\} \subseteq E(M)-B$, and let $Y_{1} \cap X_{2}=\{y\}$ where $y \in B$.

We first show that either (i) holds, or there exists an element $b_{3} \in X_{1} \cap Y_{2}$ that is not removable with respect to $B$. If $r\left(X_{1}\right)=3$, then $p$ and $q$ are removable by Lemma 3.1(ii), satisfying (i). So assume that $r\left(X_{1}\right) \geq 4$, in which case $r\left(Y_{1} \cup\left\{b_{1}\right\}\right) \leq r(M)-2$, so $\left|X_{1} \cap B\right| \geq 2$. Let $b_{3} \in X_{1} \cap B-\left\{b_{2}\right\}$, in which case $b_{3} \in Y_{2}$. If $\operatorname{si}\left(M / b_{3}\right)$ is not 3 -connected, we have one of the desired outcomes. So assume $b_{3}$ is removable. If either $p$ or $q$ is also removable, then (i) holds. Suppose neither $p$ nor $q$ is removable. Then, by Bixby's Lemma, $\operatorname{si}(M / p)$ is 3 -connected, so $\operatorname{si}(M / p) \varsubsetneqq \operatorname{co}(M \backslash q)$. It follows, by Lemma 3.3. that either $p$ is contained in a triangle other than $\left\{p, q, b_{2}\right\}$ or $q$ is contained in a triad other than $\{p, q, y\}$. If the latter, then, as in the last paragraph of 3.4.4, this leads to a contradiction. If the former, then by orthogonality and since $Y_{2} \cup\left\{b_{2}\right\}$ is closed, such a triangle is $\left\{p, y, y^{\prime}\right\}$ where $y^{\prime} \in X_{1}$ since $Y_{1} \cup\left\{b_{1}\right\}$ is closed. Furthermore $\left(y^{\prime}, y, p, q, b_{2}\right)$ is a fan ordering. By Lemma 2.12 if $y^{\prime} \in B$, then $y^{\prime}$ is not removable, and choosing $b_{3}=y^{\prime}$ we have a desired outcome. So assume $y^{\prime} \in E(M)-B$, in which case $y^{\prime}$ is removable, thereby satisfying (i).

Now, by Lemmas 2.5 and 2.6, there exists a vertical 3-separation $\left(X_{3},\left\{b_{3}\right\}, Y_{3}\right)$ such that $b_{1} \in Y_{3}$ and $Y_{3} \cup\left\{b_{3}\right\}$ is closed. By Lemma 3.2 , ( $X_{1} \cap$ $\left.X_{3}\right) \cup\left\{b_{3}\right\}$ is a rank-2 set, and if $\left|Y_{1} \cap X_{3}\right| \geq 2$, then $r\left(\left(X_{1} \cap Y_{3}\right) \cup\left\{b_{1}, b_{3}\right\}\right)=2$. But if the latter holds, then $p$ and $q$ are removable by Lemma 3.1(ii), satisfying (i). Furthermore, if $\left|\left(X_{1} \cap X_{3}\right) \cup\left\{b_{3}\right\}\right| \geq 4$, then (i) holds by Lemma 2.7. So we may assume $\left|Y_{1} \cap X_{3}\right|=1$ and $\left|X_{1} \cap X_{3}\right|=2$. Since $X_{2}$ and $X_{3}$ are triads each with two elements contained in $X_{1}$, both $y$ and the single element in $Y_{1} \cap X_{3}$ are in the coclosure of $X_{1}$. But $b_{1} \in \operatorname{cl}\left(X_{1}\right)$, so by Lemma 2.11, $Y_{1} \cap X_{3}=\{y\}$. If there exists an element $p^{\prime} \in\left(X_{1} \cap X_{3}\right) \cap B$, then (i) holds by 3.4.4. It remains to consider when $X_{1} \cap X_{3} \subseteq E(M)-B$. If $\{p, q\} \subseteq X_{3}$, 
then $p$ and $q$ are removable by Lemma 2.7, satisfying (i). Otherwise, since $Y_{3} \cup\left\{b_{3}\right\}$ is closed, $\left\{p, q, b_{2}\right\} \subseteq Y_{3}$. Let $X_{1} \cap X_{3}=\left\{p^{\prime}, q^{\prime}\right\}$. The two triads $\{p, q, y\}$ and $\left\{p^{\prime}, q^{\prime}, y\right\}$ intersect only at $y$, so $\left\{p, q, y, q^{\prime}, p^{\prime}\right\}$ is a corank-3 set. But this set contains four cobasis elements; a contradiction. So 3.4.5 holds.

We deduce that the lemma holds.

We now give two corollaries that will be of use in proving generalisations of Theorems 1.1 and 1.2 that relax the requirement that no 4-element fans are present. For the first corollary, the outcome is slightly stronger than is required.

Corollary 3.5. Let $M$ be a 3-connected matroid, where $|E(M)| \geq 3$, and let $B$ be a basis of $M$. Suppose $M$ has no Type $I$ fans relative to $B$. Then $M$ has at least three distinct elements that are removable with respect to $B$.

Proof. If every element $e \in E(M)$ is removable, then the corollary holds. Therefore, by duality, we may assume that there exists an element $b \in B$ such that $\operatorname{si}(M / b)$ is not 3-connected. By Lemmas 2.5 and 2.6, there exists a vertical 3-separation $(X,\{b\}, Y)$ of $M$ such that $Y \cup\{b\}$ is closed. By Lemma 2.6, there also exists a vertical 3-separation $\left(Y^{\prime},\{b\}, X^{\prime}\right)$ of $M$ such that $X^{\prime} \cup\{b\}$ is closed, $X \subseteq X^{\prime}$ and $Y^{\prime} \subseteq Y$. By Lemma 3.4, $X$ and $Y^{\prime}$ each contain a removable element. Thus if Lemma 3.4(i) or Lemma 3.4(iii) holds for either vertical 3-separation, the corollary holds.

We may now assume that either Lemma 3.4(ii) or Lemma 3.4(iv) holds for each of the vertical 3-separations. When Lemma 3.4(ii) holds for either vertical 3-separation, there are two removable elements $s_{1}, s_{2} \in B$ by the dual of Lemma 2.7. On the other hand, if Lemma 3.4(iv) holds for both vertical 3 -separations, again there are two removable elements $s_{1}, s_{2} \in B$ by Lemma 2.12. There exists an element $b^{*} \in E(M)-B$, as $|E(M)| \geq 3$ and $M$ is 3 -connected. If $b^{*}$ is removable, the corollary holds. Otherwise, by the dual of Lemma 2.5, there is a vertical 3-separation $\left(P,\left\{b^{*}\right\}, Q\right)$ in $M^{*}$. Next we apply Lemma 3.4 to $\left(P,\left\{b^{*}\right\}, Q\right)$. If Lemma 3.4(i) or Lemma 3.4(iii) holds, then the corollary holds, noting in the former case that there is also a removable element in $Q$ by an application of Lemma 3.4 to $\left(Q,\left\{b^{*}\right\}, P\right)$. But when Lemma 3.4(ii) or Lemma 3.4(iv) holds for $\left(P,\left\{b^{*}\right\}, Q\right)$, there exists a removable element $s_{1}^{*} \in E(M)-B$, which is distinct from $s_{1}$ and $s_{2}$. Thus the corollary holds.

Corollary 3.6. Let $M$ be a 3-connected matroid, where $|E(M)| \geq 4$, and let $B$ be a basis of $M$. Suppose $M$ has no Type I or Type II fans relative to $B$. Then $M$ has at least four distinct elements that are removable with respect to $B$.

Proof. If every element $e \in E(M)$ is removable with respect to $B$, then the corollary holds. Therefore, by duality, we may assume that there exists an element $b \in B$ such that $\operatorname{si}(M / b)$ is not 3-connected. By Lemmas 2.5 and 2.6 , there exists a vertical 3-separation $(X,\{b\}, Y)$ of $M$ such that $Y \cup\{b\}$ 
is closed. There also exists a vertical 3-separation $\left(X_{2},\{b\}, Y_{2}\right)$ of $M$ such that $X_{2} \cup\{b\}$ is closed, $X \subseteq X_{2}$ and $Y_{2} \subseteq Y$.

We can now apply Lemma 3.4 using each of the two vertical 3-separations in turn, where Lemma 3.4(iv) cannot hold since $M$ has no Type II fans. If Lemma 3.4(iii) holds for $(X,\{b\}, Y)$, then there exist distinct removable elements $s_{1} \in X$ and $s_{2}, s_{3} \in \operatorname{cl}(X)$. By an application of Lemma 3.4 to $\left(Y_{2},\{b\}, X_{2}\right)$, there is at least one removable element in $Y_{2}$, and $\left\{s_{2}, s_{3}\right\} \subseteq X_{2}$ since $X_{2} \cup\{b\}$ is closed, so the corollary holds in this case. By symmetry, we can now assume Lemma 3.4(iii) doesn't hold for either vertical 3-separation. If Lemma 3.4(i) holds for both vertical 3-separations, then clearly the corollary holds, so it remains to consider when Lemma 3.4(ii) holds for at least one of the vertical 3-separations.

Now we may assume there exists a vertical 3-separation $\left(X^{\prime},\left\{b^{\prime}\right\}, Y^{\prime}\right)$ and removable elements $s_{1} \in X^{\prime}$ and $s_{2} \in \mathrm{cl}^{*}\left(X^{\prime}\right)$, where $X^{\prime} \cup\left\{s_{2}\right\}$ is a 4element cosegment. If $b^{\prime} \in \operatorname{cl}^{*}\left(X^{\prime} \cup\left\{s_{2}\right\}\right)$, then $b^{\prime}$ is removable by the dual of Lemma 2.7; a contradiction. So $b^{\prime} \in \operatorname{cl}\left(Y^{\prime}-\left\{s_{2}\right\}\right)$ by Lemma 2.3. It follows, by Lemma 2.4, that when $r\left(Y^{\prime}-\left\{s_{2}\right\}\right) \geq 3$, the partition $\left(X^{\prime} \cup\right.$ $\left.\left\{s_{2}\right\},\left\{b^{\prime}\right\}, Y^{\prime}-\left\{s_{2}\right\}\right)$ is a vertical 3-separation. Then, by an application of Lemma 3.4 to $\left(Y^{\prime}-\left\{s_{2}\right\},\left\{b^{\prime}\right\}, X^{\prime} \cup\left\{s_{2}\right\}\right)$, the corollary holds unless there exists an element $s_{2}^{\prime} \in\left(X^{\prime} \cup\left\{s_{2}\right\}\right) \cap B$ such that $\left(Y^{\prime}-\left\{s_{2}\right\}\right) \cup\left\{s_{2}^{\prime}, b^{\prime}\right\}$ contains a 4 -element cosegment. This cosegment must contain $s_{2}^{\prime}$ and cannot contain $b^{\prime}$, by the dual of Lemma 2.7, as it is not removable. Thus, the two 4element cosegments intersect at a single element $s_{2}^{\prime}$, so the union of these two cosegments has corank three. But $s_{2}^{\prime} \in B$, so this union contains four elements of the cobasis $E(M)-B$; a contradiction. Now consider the case where $r\left(Y^{\prime}-\left\{s_{2}\right\}\right)=2$. If $\left|Y^{\prime}-\left\{s_{2}\right\}\right| \geq 3$, then, recalling $b^{\prime} \in \operatorname{cl}\left(Y^{\prime}-\left\{s_{2}\right\}\right)$, there are two elements in $Y^{\prime}-\left\{s_{2}\right\}$ that are removable by Lemma 2.7, so the corollary holds. It remains to consider when $\left|Y^{\prime}\right|=3$. Since $r(M)=4$, precisely one element of $Y^{\prime}-\left\{s_{2}\right\}$ is in $B$. But then $Y^{\prime} \cup\left\{b_{1}\right\}$ is a Type II fan; a contradiction. So the corollary holds.

\section{The Existence of Strong Elements}

In this section, we prove Theorem 1.1. The proofs of the next two lemmas are straightforward.

Lemma 4.1. Let $e$ and $f$ be distinct elements of a 3-connected matroid $M$, and suppose that $\mathrm{si}(M / e)$ is 3-connected. Then either

(i) $M / e \backslash f$ is connected, or

(ii) $\operatorname{si}(M / e) \cong U_{2,3}$ and $M$ has no triangle containing $\{e, f\}$.

Moreover, if no non-trivial parallel class of $M /$ e contains $f$, then $M / e / f$ is connected.

Lemma 4.2. Let $(X, Y)$ be a 2-separation of a connected matroid $M$ and let $N$ be a 3-connected minor of $M$. Then $\{X, Y\}$ has a member $U$ such that $|U \cap E(N)| \leq 1$. Moreover, if $u \in U$, then 
(i) $M / u$ has an $N$-minor if $M / u$ is connected, and

(ii) $M \backslash u$ has an $N$-minor if $M \backslash u$ is connected.

In the arguments that follow, we initially restrict our attention to a 3connected $N$-minor with $|E(N)| \geq 4$, so that the minor $N$ is simple and cosimple. The next lemma, whose trivial proof has been omitted, illustrates this. We handle the case where $|E(N)| \leq 3$ in Lemma 4.7 .

Lemma 4.3. Let $N$ be a 3-connected matroid such that $|E(N)| \geq 4$. If $M$ has an $N$-minor, then $\operatorname{si}(M)$ has an $N$-minor.

Lemma 4.4. Let $N$ be a 3-connected minor of a 3-connected matroid $M$ with $|E(N)| \geq 4$. Let $(X,\{z\}, Y)$ be a vertical 3-separation of $M$ such that $M / z$ has an $N$-minor, where $Y \cup\{z\}$ is closed and $|X \cap E(N)| \leq 1$. If $s \in \operatorname{cl}^{*}(X)-X$, then $\left(X^{\prime},\{z\}, Y^{\prime}\right)=(X \cup\{s\},\{z\}, Y-\{s\})$ is a vertical 3 -separation such that $Y^{\prime} \cup\{z\}$ is closed, and $\left|X^{\prime} \cap E(N)\right| \leq 1$.

Proof. Since $X$ and $X \cup\{z\}$ are exactly 3-separating in $M$, and $s \in \operatorname{cl}^{*}(X)$, it follows by Lemma 2.4 that $X^{\prime}$ and $X^{\prime} \cup\{z\}$ are 3-separating. In particular, as $r\left(Y^{\prime}\right) \geq 2$,

$$
r\left(X^{\prime}\right)+r\left(Y^{\prime} \cup\{z\}\right)=r\left(X^{\prime} \cup\{z\}\right)+r\left(Y^{\prime}\right) .
$$

So, as $z \in \operatorname{cl}\left(X^{\prime}\right)$, we have $z \in \operatorname{cl}\left(Y^{\prime}\right)$. Now, since $\left|Y^{\prime}\right| \geq 2$, the partition $\left(X^{\prime}, Y^{\prime}\right)$ is a 2-separation of $M / z$. Since $s \in \mathrm{cl}^{*}(X)$, we have $s \notin \operatorname{cl}\left(Y^{\prime}\right)$ by Lemma 2.3. Therefore, $Y^{\prime} \cup\{z\}$ is closed in $M$. By Lemma 4.2, either $\left|X^{\prime} \cap E(N)\right| \leq 1$ or $\left|Y^{\prime} \cap E(N)\right| \leq 1$. Suppose $\left|X^{\prime} \cap E(N)\right| \geq 2$. Then $|X \cap E(N)|=1$ and $|Y \cap E(N)| \leq 2$, so $|E(N)| \leq 3$; a contradiction. So $\left|X^{\prime} \cap E(N)\right| \leq 1$.

To see that $r\left(Y^{\prime}\right) \geq 3$, suppose that $r\left(Y^{\prime}\right)=2$. Then $Y^{\prime} \cup\{z\}$ is a line of at least three elements. But $|E(N)| \geq 4$, so $N$ is simple. Thus $\operatorname{si}(M / z)$ has an $N$-minor. Since $\left|X^{\prime} \cap E(N)\right| \leq 1$, the matroid $N$ is isomorphic to $U_{1,1}$ or $U_{1,2}$; a contradiction. Therefore, $r\left(Y^{\prime}\right) \geq 3$ and the lemma holds.

Let $M$ be a 3-connected matroid with a 3 -connected minor $N$. An element $x$ of $M$ is doubly $N$-labelled if $M \backslash x$ has an $N$-minor and $M / x$ has an $N$ minor.

Lemma 4.5. Let $N$ be a 3-connected minor of a 3-connected matroid $M$. Let $(X,\{z\}, Y)$ be a vertical 3-separation of $M$ such that $M / z$ has an $N$ minor, where $|X \cap E(N)| \leq 1$. If $Y \cup\{z\}$ is closed, then there is at most one element of $X$ that is not doubly $N$-labelled. Moreover, if such an element $x$ exists, then $x \in \operatorname{cl}^{*}(Y)$ and $z \in \operatorname{cl}(X-\{x\})$.

Proof. The matroid $M / z$ is the 2-sum of two matroids, $M_{X}$ and $M_{Y}$ with basepoint $z^{\prime}$ say. Note that $(M / z) \mid X=M_{X} \backslash z^{\prime}$ and $(M / z) \mid Y=M_{Y} \backslash z^{\prime}$. Let $x \in X$. Let $C_{x}$ and $C_{x}^{*}$ be a maximum-sized circuit and a maximum-sized cocircuit of $M_{X}$ containing $\left\{x, z^{\prime}\right\}$. If $\left|C_{x}\right|>2$, then $M / z / x$, and hence $M / x$, has an $N$-minor. Dually, if $\left|C_{x}^{*}\right|>2$, then $M \backslash x$ has an $N$-minor. Thus $x$ is doubly $N$-labelled unless $\left|C_{x}\right|=2$ or $\left|C_{x}^{*}\right|=2$. But if $\left|C_{x}\right|=2$, 
then $x \in \operatorname{cl}_{M / z}(Y)$, so $x \in \operatorname{cl}_{M}(Y \cup\{z\})$, contradicting the fact that $Y \cup\{z\}$ is closed. We deduce that $x$ is doubly $N$-labelled unless $\left|C_{x}^{*}\right|=2$. Moreover, $E\left(M_{X}\right)-\left\{z^{\prime}\right\}$ cannot contain distinct elements $s$ and $t$ that are not doubly $N$-labelled otherwise $\left\{z^{\prime}, s, t\right\}$ is contained in a series class of $M_{X}$ and so $\{s, t\}$ is a cocircuit of $M$; a contradiction. Thus $X$ contains at most one element that is not doubly $N$-labelled. Moreover, when such an element $x$ exists, $\left\{x, z^{\prime}\right\}$ is a cocircuit of $M_{X}$, so $x \in \mathrm{cl}^{*}{ }_{M / z}(Y)$ and $x \notin \mathrm{cl}_{M / z}(X-\{x\})$. Hence $x \in \mathrm{cl}^{*}{ }_{M}(Y)$ and $x \notin \mathrm{cl}_{M}((X-\{x\}) \cup\{z\})$. As $z \in \mathrm{cl}_{M}(X)$, it follows from the MacLane-Steinitz exchange condition that $z \in \mathrm{cl}_{M}(X-\{x\})$.

Lemma 4.6. Let $M$ be a 3-connected matroid, let $N$ be a 3-connected minor of $M$ such that $|E(N)| \geq 4$, and let $B$ be a basis of $M$ with an element $b \in B$ such that $b$ is $(N, B)$-robust, but not $(N, B)$-strong. Let $(X,\{b\}, Y)$ be a vertical 3-separation of $M$ such that $Y \cup\{b\}$ is closed and $|X \cap E(N)| \leq 1$. If $s \in X$ is removable, then $s$ is $(N, B)$-strong.

Proof. Let $s$ be a removable element of $X$. By Lemma 4.5, at most one element in $X$ is not doubly $N$-labelled, so we may assume $s$ is the only element that is not $(N, B)$-robust in $X$, in which case $s \in \mathrm{cl}^{*}(Y)$ and $b \in$ $\operatorname{cl}(X-\{s\})$. Therefore $(X-\{s\}, Y \cup\{b\})$ is a 2-separation of $M \backslash s$. If $s \in E(M)-B$, then $\operatorname{co}(M \backslash s)$ is 3 -connected, so $X-\{s\}$ is a series class in $M \backslash s$. But $b \in \operatorname{cl}(X-\{s\})$, so $\operatorname{co}(M \backslash s)$ contains a non-trivial parallel class; a contradiction. Thus $s \in B$.

Suppose $s$ and $b$ are contained in a triangle $\{s, b, q\}$ say. If $q \in X$, then $s \in \operatorname{cl}((X-\{s\}) \cup\{b\})$, so $s \notin \mathrm{cl}^{*}(Y)$ by Lemma 2.3, a contradiction. But if $q \in Y$, then $s \in \operatorname{cl}(Y \cup\{b\})=\operatorname{cl}(Y)$. Then $\{b, s\} \subseteq \operatorname{cl}(Y)-Y$ and $s \in \mathrm{cl}^{*}(Y)-Y$, contradicting Lemma 2.11. Since $s$ and $b$ are not contained in a triangle of $M$, no non-trivial parallel class of $M / s$ contains $b$, and thus by Lemma 4.1, $M / s / b$ is connected. Since $|X \cap E(N)| \leq 1$ and $s \in X$, by Lemma $4.2, M / s / b$ has an $N$-minor, and therefore $M / s$ has an $N$-minor. Thus, by Lemma 4.3, the lemma holds.

Lemma 4.7. Let $M$ be a 3-connected matroid with $|E(M)| \geq 5$, let $B$ be a basis of $M$, and suppose $M$ has a 3 -connected $N$-minor such that $|E(N)| \leq$ 3. If $s \in E(M)$ is removable, then either

(i) $s$ is an $(N, B)$-strong element, or

(ii) there exist distinct $(N, B)$-strong elements $s_{1}, s_{2} \in E(M)$, and at least one of the following holds:

(a) $r(M)=2$,

(b) $r^{*}(M)=2$,

(c) $s \in B$ and $\operatorname{si}(M / s) \cong U_{2,3}$, or

(d) $s \in E(M)-B$ and $\operatorname{co}(M \backslash s) \cong U_{1,3}$.

Proof. Since $|E(N)| \leq 3, N$ is a minor of $U_{1,3}$ or $U_{2,3}$. Thus, by duality, we may assume $N$ is a minor of $U_{2,3}$. First assume $s \in B$, in which case $\operatorname{si}(M / s)$ is 3 -connected. If $\operatorname{si}(M / s)$ has a $U_{2,3}$-minor, then (i) holds, so 
assume $\operatorname{si}(M / s)$ does not have such a minor. Then $r(M)=2$. In particular, $M \cong U_{2, n}$, where $n \geq 5$, in which case (ii) holds by Lemma 2.7.

Now assume $s \in E(M)-B$, and so $\operatorname{co}(M \backslash s)$ is 3-connected. If $\operatorname{co}(M \backslash s)$ has a circuit of at least three elements, it has a $U_{2,3}$-minor, so (i) holds. Assuming otherwise, first consider the case where $\operatorname{co}(M \backslash s)$ does not have a circuit of one or two elements. Then $\operatorname{co}(M \backslash s) \cong U_{1,1}$, so $\operatorname{si}\left(M^{*} / s\right) \cong U_{0,1}$; a contradiction.

Consider the case where $\operatorname{co}(M \backslash s)$ has a loop or a 2-circuit. If $\operatorname{co}(M \backslash s)$ has a loop, then $\operatorname{co}(M \backslash s) \cong U_{0,1}$. That is, $M^{*} \cong U_{2, n}$, where $n \geq 5$. For each element $e \in E(M), M^{*} \backslash e$ is 3 -connected and contains a $U_{1,3}$-minor. In particular, for each $e \in B, M / e$ is 3 -connected and contains a $U_{2,3^{-}}$ minor. Since $|B| \geq 2$, (ii) holds. If $\operatorname{co}(M \backslash s)$ has a 2-circuit, then either $\operatorname{co}(M \backslash s) \cong U_{1,2}$ or $\operatorname{co}(M \backslash s) \cong U_{1,3}$. If $\operatorname{co}(M \backslash s) \cong U_{1,2}$, then $\operatorname{si}\left(M^{*} / s\right) \cong$ $U_{1,2} ;$ a contradiction. Thus $\operatorname{co}(M \backslash s) \cong U_{1,3}$, that is, $\operatorname{si}\left(M^{*} / s\right) \cong U_{2,3}$.

Now, in $M^{*}$, every element lies on one of three lines intersecting at $s$ and, as $M^{*}$ is 3-connected, at least two of the lines contain three or more elements. Thus $|E(M)| \geq 6$. If one of the lines, $L$ say, containing $s$ has at least four elements, then, for each $e \in L$, we have $M^{*} \backslash e$ is 3-connected by Lemma 2.7, and it is straightforward to check that $M^{*} \backslash e$ contains a $U_{1,3}$-minor. Since at least two elements in $L$ are in $B$, we deduce that (ii) holds. Therefore each of the lines containing $s$ has at most three elements, so $|E(M)| \leq 7$. A routine check shows that (ii) holds.

Lemma 4.8. Let $M$ be a 3-connected matroid, let $N$ be a 3-connected minor of $M$ such that $|E(N)| \geq 4$, and let $B$ be a basis of $M$. Suppose $M$ has no Type I fans relative to $B$, and there exists an element $b \in B$ that is $(N, B)$ robust but not $(N, B)$-strong. Let $(X,\{b\}, Y)$ be a vertical 3 -separation of $M$ such that $|X \cap E(N)| \leq 1$. Then one of the following holds:

(i) there exist distinct $(N, B)$-strong elements $s_{1}, s_{2} \in X$, or

(ii) there exist distinct $(N, B)$-strong elements $s_{1} \in X$ and $s_{2} \in \mathrm{cl}^{*}(X) \cap$ $B$, or

(iii) there exist distinct $(N, B)$-strong elements $s_{1} \in X$ and $s_{2}, s_{3} \in$ $\operatorname{cl}(X) \cap(E(M)-B)$, or

(iv) $X \cup\{b\}$ contains a Type II fan $F$ and an $(N, B)$-strong element $s \in F \cap B$.

Proof. By Lemma 2.6, there exists a vertical 3-separation $\left(X^{\prime},\{b\}, Y^{\prime}\right)$ such that $Y^{\prime} \cup\{b\}$ is closed and $X^{\prime} \subseteq X$. If the lemma holds for the vertical 3 -separation $\left(X^{\prime},\{b\}, Y^{\prime}\right)$, then clearly it holds for the vertical 3-separation $(X,\{b\}, Y)$; so we may assume that $Y \cup\{b\}$ is closed. If $X \cup\{b\}$ contains a Type II fan $F$, then, by Lemma 2.12, there exists a removable element in $F \cap B$. By Lemma 4.6, such a removable element is $(N, B)$-strong, satisfying (iv).

Now assume $X \cup\{b\}$ does not contain a Type II fan. By Lemma 3.4, there is an element $s_{1} \in X$ and either a distinct element $s_{2} \in X$, a distinct element $s_{2} \in \mathrm{cl}^{*}(X) \cap B$, or distinct elements $s_{2}, s_{3} \in \operatorname{cl}(X) \cap(E(M)-B)$, where 
each $s_{i}$ is removable with respect to $B$ for $i \in\{1,2,3\}$. By Lemma 4.6. the element $s_{1}$ is $(N, B)$-strong, and if $s_{i} \in X$ for $i \in\{2,3\}$, then $s_{i}$ is also $(N, B)$-strong, in which case (i) holds. Consider the case where $s_{2} \in$ $\mathrm{cl}^{*}(X) \cap B$. By Lemma 4.4, $\left(X \cup\left\{s_{2}\right\},\{b\}, Y-\left\{s_{2}\right\}\right)$ is a vertical 3-separation where $\left|\left(X \cup\left\{s_{2}\right\}\right) \cap E(N)\right| \leq 1$ and $\left(Y-\left\{s_{2}\right\}\right) \cup\{b\}$ is closed. By Lemma 4.6, $s_{2}$ is $(N, B)$-strong, so (ii) holds. It remains to consider the case where $s_{2}, s_{3} \in(\operatorname{cl}(X)-X) \cap(E(M)-B)$. Now, $\left\{b, s_{2}, s_{3}\right\} \subseteq \operatorname{cl}(X) \cap \operatorname{cl}(Y)$, and, by submodularity, $r(\operatorname{cl}(X) \cap \operatorname{cl}(Y)) \leq 2$, so $r\left(\left\{b, s_{2}, s_{3}\right\}\right)=2$. The matroid $M / b$ has an $N$-minor, and $N$ has no 2-circuits, but $s_{2}$ and $s_{3}$ are in parallel in $M / b$. It follows that $M / b \backslash s_{2}$ and $M / b \backslash s_{3}$ have $N$-minors, so $s_{2}$ and $s_{3}$ are $(N, B)$-strong by Lemma 4.3 , satisfying (iii). We deduce that the lemma holds.

We are now in a position where we can prove Theorem 1.1. In particular, it is a special case of the next theorem.

Theorem 4.9. Let $M$ be a 3-connected matroid such that $|E(M)| \geq 5$, let $N$ be a 3-connected minor of $M$, and let $B$ be a basis of $M$. Suppose $M$ has no Type I fans relative to $B$. If $M$ has at least two distinct $(N, B)$-robust elements, then $M$ has at least two distinct $(N, B)$-strong elements.

Proof. Since $M$ has no Type I fans, $M$ has at least three removable elements by Corollary 3.5. If $|E(N)| \leq 3$, then it follows, by Lemma 4.7, that the theorem holds. So assume that $|E(N)| \geq 4$. Let $p_{1}$ and $p_{2}$ be distinct $(N, B)$-robust elements. If $p_{1}$ and $p_{2}$ are both $(N, B)$-strong elements, then the theorem holds; so assume otherwise. By duality, we may assume that $p_{1}$, say, is not $(N, B)$-strong, and is a member of $B$. Since $\operatorname{si}\left(M / p_{1}\right)$ is not 3-connected, by Lemmas 2.5 and 4.2 there exists a vertical 3 -separation $\left(X,\left\{p_{1}\right\}, Y\right)$ such that $|X \cap E(N)| \leq 1$. Then, by Lemma 4.8, the theorem holds unless $X \cup\left\{p_{1}\right\}$ contains a Type II fan $F$.

Let $\left(f_{1}, f_{2}, f_{3}, f_{4}\right)$ be a fan ordering of $F$ such that $\left\{f_{2}, f_{3}, f_{4}\right\} \subseteq X$, $f_{2} \in E(M)-B$, and $f_{4} \in B$ is $(N, B)$-strong. We next show that $\operatorname{co}\left(M \backslash f_{2}\right)$ has an $N$-minor. We may assume, by Lemma 2.6, that $Y \cup\left\{p_{1}\right\}$ is closed. By Lemma 4.5, at most one element of $\left\{f_{2}, f_{3}, f_{4}\right\}$ is not doubly $N$-labelled. If $f_{2}$ is doubly $N$-labelled, then $\operatorname{co}\left(M \backslash f_{2}\right)$ has an $N$-minor. If $f_{2}$ is not doubly $N$-labelled, then $f_{3}$ is doubly $N$-labelled and so $\operatorname{si}\left(M / f_{3}\right) \cong \operatorname{si}\left(M / f_{3} \backslash f_{2}\right)$ has an $N$-minor. But then $\operatorname{co}\left(M \backslash f_{2}\right)$ again has an $N$-minor. If $\operatorname{co}\left(M \backslash f_{2}\right)$ is 3 -connected, then the theorem holds. So assume $f_{2}$ is not strong, where $f_{2}$ is a member of the basis $E(M)-B$ of $M^{*}$. By Lemmas 2.5 and 4.2 there exists a vertical 3-separation $\left(P,\left\{f_{2}\right\}, Q\right)$ in $M^{*}$ such that $|P \cap E(N)| \leq 1$. By Lemma 4.8 , either $M^{*}$ has at least two $\left(N^{*}, E(M)-B\right)$-strong elements, in which case the theorem holds, or $M^{*}$ has a Type II fan $F^{*}$ containing an $\left(N^{*}, E(M)-B\right)$-strong element. But, in the latter case, the $\left(N^{*}, E(M)-B\right)$ strong element in $M^{*}$ is an $(N, B)$-strong element in $M$, and is a member of the basis of $M^{*}$; that is, it is a member of $E(M)-B$. Since $f_{4} \in B$ is also $(N, B)$-strong, the theorem holds. 


\section{Two ExAmples}

In this section we give two examples to illustrate that Theorem 4.9 is best possible in two senses; firstly, that it is a necessary condition that the matroid have at least two robust elements, and secondly, it is necessary the matroid does not have a Type I fan.

For the first example, we describe a 3-connected matroid $M_{2}$ with a basis $B$ and an $N$-minor, where both the size of the ground set of $M_{2}$ and the difference in sizes of the ground sets of $M_{2}$ and $N$ can be made arbitrarily large, yet $M_{2}$ has only a single $(N, B)$-robust element.

Let $M$ and $M^{+}$be matroids such that $M=M^{+} \backslash e$ where $e \in E\left(M^{+}\right)$. Recall that $M^{+}$is the free extension of $M$ if $M^{+}$has the same rank as $M$ and every circuit of $M^{+}$containing $e$ is spanning. In what follows, the $N$ minor is the Fano matroid $F_{7}$, but any sufficiently structured matroid would do. Our example is of a similar nature as that given by Oxley et al. [4, to demonstrate the tightness of their result. As in that example, we make use of the following lemma.

Lemma 5.1. Let $M^{+}$be a free extension of $M$.

(i) If an element a of $M$ is not a coloop of $M$, then $M^{+} \backslash a$ is a free extension of $M \backslash a$ and $M^{+} / a$ is a free extension of $M / a$.

(ii) If $M$ has no $F_{7}$-minor, then $M^{+}$has no $F_{7}$-minor.

Let $k$ be a positive integer and let $M_{1}$ be a matroid obtained by coextending $F_{7} k$ times such that $r\left(M_{1}\right)=k+3$ and $M_{1}$ is 3 -connected. One way to obtain such a matroid $M_{1}$ is to freely coextend $F_{7} k$ times. Note that $r^{*}\left(M_{1}\right)=r^{*}\left(F_{7}\right)$ so that, for all $a \in E\left(M_{1}\right)$, the matroid $M_{1} \backslash a$ does not have an $F_{7}$-minor. Let $M_{2}$ be the matroid obtained by freely extending $M_{1} k+2$ times.

Let $x \in E\left(M_{1}\right)-E\left(F_{7}\right)$ and let $B=\left(E\left(M_{2}\right)-E\left(M_{1}\right)\right) \cup\{x\}$. We show that $B$ is a basis of $M_{2}$. Suppose it is not. Then, since $|B|=k+3$, the set $B$ contains a circuit $C$. If $C$ contains an element in $E\left(M_{2}\right)-E\left(M_{1}\right)$, then since every circuit containing this element is spanning, $C$ is spanning, and thus $|C|=k+4$; a contradiction. But then $C \subseteq E\left(M_{1}\right)$, and so $C=\{x\}$; a contradiction. So $B$ is indeed a basis of $M_{2}$.

We can contract $x$ and retain the $F_{7}$-minor, since $x \in E\left(M_{1}\right)-E\left(F_{7}\right)$ and $M_{2} /\left(E\left(M_{1}\right)-E\left(F_{7}\right)\right) \backslash\left(E\left(M_{2}\right)-E\left(M_{1}\right)\right)=F_{7}$. Thus $x$ is $\left(F_{7}, B\right)$ robust. However, as $M_{1} \backslash a$ has no $F_{7}$-minor for all $a \in E\left(M_{1}\right)$, it follows by Lemma 5.1 that $M_{2} \backslash d$ has no $F_{7}$-minor for all $d \in E\left(M_{2}\right)-B$. Now let $b \in B-\{x\}$. To obtain a 7-element rank-3 minor of $M_{2} / b$, we must delete an element in $E\left(M_{1}\right)-\{x\}$. But we have seen that if we delete such an element then the matroid has no $F_{7}$-minor. Thus $M_{2} / b$ has no $F_{7}$-minor for all $b \in B-\{x\}$. We conclude that $M_{2}$ has only a single $\left(F_{7}, B\right)$-robust element.

For the second example, we describe a 3-connected matroid $M_{2}$ with an $N$-minor, a fixed basis $B$, and containing a Type I fan relative to $B$. 
However, even though $M_{2}$ has more than two $(N, B)$-robust elements, it has no $(N, B)$-strong elements. Again, we base our argument on the Fano matroid $F_{7}$, but any sufficiently structured matroid with a 3 -point line would work.

Let $L=\left\{x_{1}, x_{2}, x_{3}\right\}$ be a 3 -point line of $F_{7}$. Add three elements $x_{1}^{\prime}, x_{2}^{\prime}$, and $x_{3}^{\prime}$, in parallel with $x_{1}, x_{2}$ and $x_{3}$, respectively. Perform a $\Delta-Y$ exchange on $\left\{x_{1}^{\prime}, x_{2}^{\prime}, x_{3}^{\prime}\right\}$. In the resulting matroid $M_{1}$, let $\left\{x_{1}, y_{2}, y_{3}\right\},\left\{y_{1}, x_{2}, y_{3}\right\}$ and $\left\{y_{1}, y_{2}, x_{3}\right\}$ be distinct lines, where $E\left(M_{1}\right)-E\left(F_{7}\right)=\left\{y_{1}, y_{2}, y_{3}\right\}$. Add an element $x_{1}^{\prime \prime}$ in parallel with $x_{1}$, and an element $y_{2}^{\prime}$ in parallel with $y_{2}$. Perform a $\Delta-Y$ exchange on $\left\{x_{1}^{\prime \prime}, y_{2}^{\prime}, y_{3}\right\}$. It is straightforward to show that the resulting matroid $M_{2}$ is 3 -connected. Let $E\left(M_{2}\right)-E\left(M_{1}\right)=\left\{z_{1}, z_{2}, z_{3}\right\}$, such that $\left\{z_{1}, y_{2}, z_{3}\right\}$ and $\left\{x_{1}, z_{2}, z_{3}\right\}$ are distinct 3 -point lines. $M_{2}$ has a 7 element fan with fan ordering $\left(x_{3}, y_{1}, y_{2}, z_{1}, z_{3}, z_{2}, x_{1}\right)$. For clarity, we relabel these elements such that the same fan ordering is $\left(f_{1}, f_{2}, f_{3}, f_{4}, f_{5}, f_{6}, f_{7}\right)$.

Let $B$ be a basis of $M$ such that $f_{i} \in B$ when $i$ is odd, and $f_{i} \in E(M)-B$ when $i$ is even. Note that $\left\{f_{3}, f_{4}, f_{5}, f_{6}\right\}$ is a Type I fan relative to $B$, for example. Evidently, $f_{i}$ is an $\left(F_{7}, B\right)$-robust element for all $i \in\{2,3, \ldots, 6\}$, but every other element of $M$ is not $\left(F_{7}, B\right)$-robust. By Lemma 2.12 , none of the elements $\left\{f_{2}, f_{3}, \ldots, f_{6}\right\}$ are $\left(F_{7}, B\right)$-strong. Thus, even though $M$ has at least two distinct $\left(F_{7}, B\right)$-robust elements, $M$ has no $\left(F_{7}, B\right)$-strong elements.

\section{Matroids with a Minimum Number of Strong Elements}

In this section, we consider the structure of matroids that have the minimum number of strong elements. In particular, we establish Theorem 1.2 .

A path of 3-separations in a matroid $M$ is an ordered partition $\left(P_{0}, P_{1}, \ldots, P_{k}\right)$ of $E(M)$ with the property that $\lambda\left(P_{0} \cup P_{1} \cup \cdots \cup P_{i}\right)=2$ for all $i \in\{0,1, \ldots, k-1\}$. Note that a vertical 3 -separation $(X,\{z\}, Y)$ is a path of 3 -separations. The following lemma is elementary.

Lemma 6.1. A partition $(X, Y)$ of a matroid $M$ such that $|X|,|Y| \geq 2$ is a sequential 3-separation if and only if, for some $U \in\{X, Y\}$, there is a path of 3-separations $\left(P_{0}, P_{1}, \ldots, P_{k}, U\right)$ in $M$ such that $\left|P_{0}\right|=2$, and $\left|P_{i}\right|=1$ for all $i \in\{1,2, \ldots, k\}$.

We also make use of the following result [9, Corollary 5.3]:

Lemma 6.2. Let $\mathbb{P}=\left(P_{0}, \ldots, P_{r}\right)$ be a path of 3-separations in a matroid M. Suppose $i \in\{1,2, \ldots, r-1\}, e \in P_{i}$, and that there exists a path of 3-separations $(X,\{e\}, Y)$ in $M$ with $P_{0} \subseteq X$ and $P_{r} \subseteq Y$. Then $\mathbb{P}$ refines to a path of 3 -separations $\left(P_{0}, \ldots, P_{i-1}, P_{i}^{\prime},\{e\}, P_{i}^{\prime \prime}, P_{i+1}, \ldots, P_{r}\right)$ where $P_{i}^{\prime} \cup$ $\{e\} \cup P_{i}^{\prime \prime}=P_{i}$.

Lemma 6.3. Let $M$ be a 3-connected matroid with ground set $E$, and let $s_{1}$ and $s_{2}$ be distinct elements of $M$. Let $Z$ be a subset of $E-\left\{s_{1}, s_{2}\right\}$ where $\left|E-\left(Z \cup\left\{s_{1}, s_{2}\right\}\right)\right| \geq 2$ and, for each $z \in Z$, there exists a path of 
3-separations $\left(X_{z},\{z\}, Y_{z}\right)$ in $M$ such that $\left\{s_{1}, s_{2}\right\} \subseteq X_{z}$ and $X_{z} \subseteq Z \cup$ $\left\{s_{1}, s_{2}\right\}$. Then

$$
\left(\left\{s_{1}, s_{2}\right\},\left\{z_{1}\right\},\left\{z_{2}\right\}, \ldots,\left\{z_{k}\right\}, E-\left(Z \cup\left\{s_{1}, s_{2}\right\}\right)\right)
$$

is a path of 3-separations in $M$.

Proof. Let $S=\left\{s_{1}, s_{2}\right\}$. If $Z$ is empty, the result holds immediately. So assume that $Z$ is non-empty. Let $Z=\left\{z_{1}, z_{2}, \ldots, z_{k}\right\}$ be a subset of $E-S$ as described in the statement of the lemma. Now, for all $i \in\{1,2, \ldots, k\}$, the tuple $\left(X_{z_{i}},\left\{z_{i}\right\}, Y_{z_{i}}\right)$ is a path of 3 -separations in $M$. Since $S \subseteq X_{z_{i}}$ for all $i$, it follows that $S \subseteq E-\left(Y_{z_{1}} \cup Y_{z_{2}} \cup \cdots \cup Y_{z_{k}}\right)$. In particular, $\left|E-\left(Y_{z_{1}} \cup Y_{z_{2}} \cup \cdots \cup Y_{z_{k}}\right)\right| \geq 2$ and so, by Corollary 2.2 , $Y_{z_{1}} \cap Y_{z_{2}} \cap \cdots \cap Y_{z_{k}}=$ $E-(Z \cup S)$ is 3-separating. Since $|S| \geq 2$ and $|E-(Z \cup S)| \geq 2$, the partition $(S, Z, E-(Z \cup S))$ is a path of 3 -separations in $M$. By repeatedly applying Lemma 6.2 , we deduce that the lemma holds.

Lemma 6.4. Let $M$ be a 3-connected matroid, let $B$ be a basis of $M$, and let $N$ be a 3-connected minor of $M$ such that $|E(N)| \geq 4$. Suppose $M$ has no Type $I$ or Type II fans relative to $B$, and suppose there are precisely two distinct elements $s_{1}$ and $s_{2}$ that are $(N, B)$-strong in $M$. Let $P$ denote the set of $(N, B)$-robust elements of $M$. Then, for every $z \in P-\left\{s_{1}, s_{2}\right\}$, there exists a path of 3-separations $\left(X_{z},\{z\}, Y_{z}\right)$ such that $\left\{s_{1}, s_{2}\right\} \subseteq X_{z}$ and $X_{z} \subseteq P$.

Proof. Let $S=\left\{s_{1}, s_{2}\right\}$. Consider an element $z \in P-S$. By duality, we may assume that $z \in B$. First we show the following:

6.4.1. There exists a vertical 3-separation $\left(X^{\prime},\{z\}, Y^{\prime}\right)$ such that $s_{1}, s_{2} \in$ $X^{\prime},\left|X^{\prime} \cap E(N)\right| \leq 1$, and $Y^{\prime} \cup\{z\}$ is closed.

Since $\operatorname{si}(M / z)$ is not 3-connected, it follows by Lemmas 2.5 and 2.6 that there exists a vertical 3-separation $(X,\{z\}, Y)$ such that $Y \cup\{z\}$ is closed. By Lemma 4.2, either $|X \cap E(N)| \leq 1$ or $|Y \cap E(N)| \leq 1$. If the latter, then by applying Lemma 2.6 and relabelling, we can obtain a vertical 3-separation $(X,\{z\}, Y)$ such that $Y \cup\{z\}$ is closed and $|X \cap E(N)| \leq 1$. By Lemma 3.4. there is an element $s_{1} \in X$ and either a distinct element $s_{2} \in \operatorname{cl}^{*}(X) \cap B$, or distinct elements $s_{2}, s_{3} \in \operatorname{cl}(X) \cap(E(M)-B)$, where each $s_{i}$ is removable with respect to $B$ for $i \in\{1,2,3\}$.

In the first case, there exists a vertical 3-separation $\left(X^{\prime},\{z\}, Y^{\prime}\right)$ such that $\left\{s_{1}, s_{2}\right\} \subseteq X^{\prime},\left|X^{\prime} \cap E(N)\right| \leq 1$, and $Y^{\prime} \cup\{z\}$ is closed by Lemma 4.4. By Lemma 4.6, $s_{1}$ and $s_{2}$ are $(N, B)$-strong, satisfying 6.4.1. In the second case, as $M$ has precisely two $(N, B)$-strong elements, it follows by Lemma 4.6 that $\left\{s_{2}, s_{3}\right\} \nsubseteq X$. If exactly one of $s_{2}$ and $s_{3}$ is in $X$, then this element is strong, and $s_{1}$ and this element satisfies 6.4.1. So we can assume $\left\{s_{2}, s_{3}\right\} \subseteq \operatorname{cl}(X)-$ $X$. It follows that $\left\{z, s_{2}, s_{3}\right\} \subseteq \operatorname{cl}(X) \cap \operatorname{cl}(Y)$, and so $r\left(\left\{z, s_{2}, s_{3}\right\}\right)=2$. Recall that $\left\{s_{2}, s_{3}\right\} \subseteq E(M)-B$, the matroid $M / z$ has an $N$-minor, and $N$ has no 2-circuits. Now $s_{2}$ and $s_{3}$ are parallel in $M / z$, thus $M / z \backslash s_{2}$ and $M / z \backslash s_{3}$ have $N$-minors, and, by Lemma $4.3, s_{2}$ and $s_{3}$ are $(N, B)$-strong. 
But $s_{1}$ is also $(N, B)$-strong by Lemma 4.6 , a contradiction. We deduce that 6.4.1 holds.

Now, by Lemma 4.5, at most one element in $X^{\prime}$ is not doubly $N$-labelled, and if such an element $x$ exists, then $x \in \mathrm{cl}^{*}\left(Y^{\prime}\right)$. Suppose such an $x$ exists, and $x$ is not $(N, B)$-robust. By Lemma 2.4. $Y^{\prime} \cup\{x\}$ and $Y^{\prime} \cup\{x, z\}$ are 3 -separating. Since $\left|X^{\prime}\right| \geq 3$, these 3 -separating sets are exact. It follows that $\left(X^{\prime \prime},\{z\}, Y^{\prime \prime}\right)=\left(X^{\prime}-\{x\},\{z\}, Y^{\prime} \cup\{x\}\right)$ is a path of 3-separations such that $S \subseteq X^{\prime \prime}$ and $X^{\prime \prime} \subseteq P$. Otherwise, when no such $x$ exists or $x$ is $(N, B)$-robust, every element in $X$ is robust, so $X^{\prime} \subseteq P$. This completes the proof of the lemma.

Theorem 1.2 is an immediate consequence of the next theorem.

Theorem 6.5. Let $M$ be a 3-connected matroid such that $|E(M)| \geq 5$, let $B$ be a basis of $M$, and let $N$ be a 3-connected minor of $M$. Suppose $M$ has no Type I or Type II fans relative to $B$, and let $P$ denote the set of $(N, B)$ robust elements of $M$. If $M$ has precisely two $(N, B)$-strong elements, then $(P, E(M)-P)$ is a sequential 3-separation.

Proof. Let $S=\left\{s_{1}, s_{2}\right\}$ denote the set of $(N, B)$-strong elements of $M$. First suppose that $|E(N)| \geq 4$. By Lemma 6.4 for every $z \in P-S$ there exists a path of 3 -separations $\left(X_{z},\{z\}, Y_{z}\right)$ such that $S \subseteq X_{z}$ and $X_{z} \subseteq P$. By Corollary 3.6, $M$ has at least four elements that are removable with respect to $B$. However, only two of these elements are $(N, B)$-strong, and so $\mid E(M)-$ $P \mid \geq 2$. It follows, by Lemma 6.3, that $\left(S,\left\{z_{1}\right\},\left\{z_{2}\right\}, \ldots,\left\{z_{k}\right\}, E(M)-P\right)$ is a path of 3 -separations, where $P-S=\left\{z_{1}, z_{2}, \ldots, z_{k}\right\}$. Thus, by Lemma 6.1. $(P, E(M)-P)$ is a sequential 3-separation.

It remains to consider when $|E(N)| \leq 3$. We show that, in this case, $M$ has more than two $(N, B)$-strong elements, thereby resulting in a contradiction. If $r(M) \leq 2$, then $M \cong U_{2, n}$ where $n \geq 5$, and it is easily checked that $M$ has at least three $(N, B)$-strong elements. Thus, by duality, we can assume that $r(M) \geq 3$ and $r^{*}(M) \geq 3$. By Lemma 4.7 and Corollary 3.6. either

(i) there are at least three $(N, B)$-strong elements, or

(ii) up to duality, there is a removable element $s \in B$ and $\operatorname{si}(M / s) \cong U_{2,3}$.

If (i) holds, then we obtain a contradiction. So assume (ii) holds. Then $M$ consists of three lines that intersect at the element $s$, at least two of which contain three or more elements, since $M$ is 3-connected. If one of these lines, $L$ say, consists of at least four elements, then, by Lemma 2.7. $L$ contains two elements in $E(M)-B$ that are removable with respect to $B$, and retain a $U_{1,3^{-}}$or a $U_{2,3}$-minor. Furthermore, there exists at least one element $b \in B \cap(E(M)-L)$ such that $\operatorname{si}(M / b) \cong U_{2, n}$ for some $n \geq 4$. Thus, $b$ is $(N, B)$-strong, so $M$ has more than two $(N, B)$-strong elements; a contradiction. If $|E(M)| \in\{6,7\}$, then it is straightforward to check that $M$ has at least three $(N, B)$-strong elements for every $B$ and $N$ such that $|E(N)| \leq 3$; a contradiction. This completes the proof of the theorem. 


\section{ACKNOWLEDGEMENTS}

We thank the anonymous referees for their particularly careful reading of the paper, and valuable comments.

\section{REFERENCES}

[1] R.E. Bixby, A simple theorem on 3-connectivity, Linear Algebra Appl. 45 (1982) 123-126.

[2] J. Oxley, Matroid Theory, second ed., Oxford University Press, New York, 2011.

[3] J. Oxley, C. Semple, G. Whittle, Wild triangles in 3-connected matroids, J. Combin. Theory Ser. B 98(2) (2008) 291-323.

[4] J. Oxley, C. Semple, G. Whittle, Maintaining 3-connectivity relative to a fixed basis, Adv. in Appl. Math. 41 (2008) 1-9.

[5] J. Oxley, H. Wu, On the structure of 3-connected matroids and graphs, European J. Combin. 21 (2000) 667-688.

[6] P.D. Seymour, Decomposition of regular matroids, J. Combin. Theory Ser. B 28 (1980) 305-359.

[7] W.T. Tutte, Connectivity in matroids, Canad. J. Math. 18 (1966) 1301-1324.

[8] G. Whittle, Stabilizers of classes of representable matroids, J. Combin. Theory Ser. B 77 (1999) 39-72.

[9] G. Whittle, A. Williams, On maintaining matroid 3-connectivity relative to a fixed basis, European J. Combin., in press.

Department of Mathematics and Statistics, University of Canterbury, Christchurch, New Zealand

E-mail address: nick.brettell@pg.canterbury.ac.nz

Department of Mathematics and Statistics, University of Canterbury, Christchurch, New ZeAland

E-mail address: charles.semple@canterbury.ac.nz 